\title{
Cell Origin Subtypes Predict Outcomes in Localized-Stage Diffuse Large B-Cell Lymphoma Treated with Curative Radiotherapy
}

\author{
Yuko Watanabe ${ }^{1}$, Hiroaki Suefuji ${ }^{1}$, Etsuyo Ogo ${ }^{1}$, Kensaku Sato $^{2}$, Tadashi Nakashima ${ }^{3}$, \\ Takashi Okamura ${ }^{4}$, Koichi Ohshima ${ }^{5}$, Naofumi Hayabuchi ${ }^{1}$ \\ ${ }^{1}$ Department of Radiology, Kurume University School of Medicine, Kurume, Japan; ${ }^{2}$ Biostastics Center, Kurume University School \\ of Medicine, Kurume, Japan; ${ }^{3}$ Department of Otolaryngology, Head and Neck Surgery, Kurume University School of Medicine, \\ Kurume, Japan; ${ }^{4}$ Department of Hematology and Oncology, Kurume University School of Medicine, Kurume, Japan; ${ }^{5}$ Department of \\ Pathology, Kurume University School of Medicine, Kurume, Japan. \\ Email: sufg@med.kurume-u.ac.jp
}

Received November $21^{\text {st }}, 2012$; revised January $23^{\text {rd }}, 2013$; accepted January $31^{\text {st }}, 2013$

\begin{abstract}
Diffuse large B-cell lymphoma (DLBCL) is regarded as a heterogeneous group of lymphomas. The aims of this study were to determine the clinical significance and prognostic value of different immunophenotypic profiles in localizedstage head and neck DLBCL treated with curative radiotherapy. We included 102 localized-stage head and neck DLBCL patients in this study. We classified DLBCL patients into germinal center B-cell (GCB) and non-GCB groups by immunohistochemical analysis. Statistical analysis was used to correlate the GCB and non-GCB subgroups, CD5 and Ki67 expression, B-ALPS (a modified International Prognostic Index for early stage lymphoma), chemotherapy regimen, and sex. Multivariate analysis was performed using the Cox proportional hazard regression model to compare cause-specific survival (CSS) and relapse-free rate (RFR) distributions. The cell of origin classification (GCB or nonGCB subtypes) was an independent predictor of CSS $(p=0.040)$ and RFR $(p=0.023)$. In the non-GCB group, chemotherapy with rituximab was an independent predictor of CSS. In conclusion, this study shows that the prognosis of the non-GCB group was significantly poorer than that of the GCB group, and that rituximab improved CSS in localizedstage head and neck DLBCL, especially in the non-GCB group.
\end{abstract}

Keywords: GCB; Non-GCB; DLBCL; Localized-Stage; Prognosis

\section{Introduction}

Diffuse large B-cell lymphoma (DLBCL) is the most common type of non-Hodgkin lymphoma (NHL) and accounts for $30 \%$ to $40 \%$ of newly diagnosed NHL cases $[1,2]$. DLBCL is regarded as a heterogeneous group of lymphomas in terms of surface markers, histology, and clinical features [3]. The International Prognostic Index (IPI) has remained the most important independent prognostic factor in survival analysis and has been widely used to predict the prognosis of NHL patients [4]. Differences in outcomes between groups of patients with DLBCL, defined by the 5 IPI clinical parameters (extranodal involvement, age, lactate dehydrogenase [LDH] level, performance status [PS], and stage), reflect a mixture of underlying biological and genetic differences that affect the patient's responsiveness to therapies. Most stud-

${ }^{*}$ Corresponding author. ies regarding the heterogeneity of DLBCL have focused on the morphologic features, individual protein expression, or molecular alterations [5-9]. Combining these biomarkers may add to the prognostic information gleaned from the clinical variables in the clinical prognostic index.

The expression of individual antigens related to different stages of B-cell differentiation, including CD10, BCL6, MUM1/IRF4, and CD5 may help to define groups of tumors with different clinical and pathological characteristics [10-13]. The cell of origin classification based on gene expression profiling has shown that a germinal center B-cell (GCB) profile predicts a better survival when compared to a non-GCB profile, among DLBCL patients treated by chemotherapy with or without rituximab $[5,6,9,14]$. This DLBCL subclassification (GCB or non-GCB) has been translated to paraffin-embedded tissues by combined immunostaining of CD10, BCL6, and 
MUM1/IRF4 using the Hans algorithm [15]. Various studies have shown that this classification on the basis of the cell of origin is an important independent prognostic factor for patients who are treated with CHOP- (cyclophosphamide, doxorubicin, vincristine, and prednisone) like chemotherapy [15-17]. GCB and non-GCB phenotypes are predictors of outcome in DLBCL and can be used to stratify chemotherapy-treated patients into lowand high-risk groups. Some reports have shown that survival outcome for treatment with CHOP-like chemotherapy tended to be much better in patients with the GCB subtype than in patients with the non-GCB subtype [1519]. In the rituximab era, some reports have suggested that the prognostic value for the distinction of the GCB and non-GCB was loss for patients treated with R-CHOP $[18,19]$. On the other hand, it has been reported that a difference in survival between GCB and non-GCB subtypes was still present [20]. Ki67 is a nuclear antigen expressed in cells that are proliferating, and has been considered a useful prognostic index in various malignancies, including DLBCL [21-24]. It has been reported that prognosis is likely to be poorest for patients with a non-GCB phenotype and high Ki67 expression when compared to the other subgroups. In addition, elevated Ki67 expression indicated a limited survival benefit of R-CHOP (CHOP with rituximab) therapy for non-GCB DLBCL patients [25]. CD5-positive (CD5+) DLBCL is a subtype of DL$\mathrm{BCL}$ and it has been reported that its clinical outcome is poorer than CD5-negative (CD5-) DLBCL [12].

About the effect of radiotherapy, it has been reported that no difference was found in the survival rates between patients receiving radiotherapy alone and those receiving radiotherapy and CHOP-like chemotherapy [26]. It has been also suggested localized oral NHL with no ulceration and intermediate histologic features may be successfully treated with radiotherapy alone [27]. However, no report has been found that evaluates the difference of effect between radiotherapy alone and the combination of radiotherapy and chemotherapy with rituximab in NHL.

It is important to identify at diagnosis, patients who may need more aggressive therapies; moreover, there is a need for biomarkers that accurately predict the outcome for these patients. However, thus far, no study has been published regarding the relationship between classification based on the cell of origin and prognosis in localized-stage head and neck DLBCL which indicates curative radiotherapy. Therefore, the aims of this study were to determine the clinical significance and prognostic value of different immunophenotypic profiles, and to investigate the possible relationship of these groups of tumors with different oncogenic and proliferative markers, in localized-stage head and neck DLBCL treated with cura- tive radiotherapy, with or without chemotherapy.

\section{Materials and Methods}

\subsection{Patients}

The study population consisted of 133 patients with localized-stage head and neck DLBCL who were treated at the Kurume University Hospital between January 2000 and December 2010. All patients received radiotherapy, with or without chemotherapy (including immunochemotherapy). Basically, radiotherapy and chemotherapy were received, but chemotherapy could not be added because of the condition of the patients, taking into account factors such as worse PS, high age, and systemic complications; also, some patients refused chemotherapy.

All specimens for histologic and immunophenotypic studies were obtained at the initial diagnosis. However, 31 patients were excluded for one of the following reasons: 1) specimens were inadequate for immunostaining or 2) paraffin blocks were unavailable for immunostaining because the patients were referred from other institutions. The remaining 102 DLBCL patients were included in the present study.

Radiotherapy was performed using involved field and the median total dose of radiotherapy was $30.6 \mathrm{~Gy}$ (range, $27 \mathrm{~Gy}-61 \mathrm{~Gy}$, over $95 \%$ of patients received under 40.6 Gy). The basic dose of radiotherapy was $30.6 \mathrm{~Gy}$ in radiotherapy with chemotherapy cases, and 40.6 Gy in radiotherapy alone cases. The primary sites for these patients were Waldeyer's ring (50 cases), lymph node (LN) ( 20 cases), thyroid (10 cases), paranasal sinus (10 cases), nasal cavity ( 5 cases), nasopharynx ( 3 cases), hypopharynx ( 2 cases), and oral cavity ( 2 cases). As mentioned earlier, the IPI has been widely used to predict the prognosis of NHL patients [4]. Stages were originally grouped as I or II vs III or IV, but this can be modified to I vs II when analyzing early-stage NHL. Oguchi et al. introduced a new prognostic index, B-ALPS (tumor bulk, age, LDH level, PS, and stage,), which included tumor bulk $(\geq 6 \mathrm{~cm}$ vs $<6 \mathrm{~cm}$ ) instead of the amount of extranodal involvement [28]. Hayabuchi et al. reported that B-ALPS appears to be a better index for Stage I and II B-cell NHL of the head and neck [26]. Therefore, we have used BALPS instead of IPI as the clinical index in this study.

Disease remission was defined as absence of disease for at least 1 month after cessation of the last treatment regimen, as assessed by laboratory and imaging studies and physical examinations. Disease relapses were defined as disease recurring at least 1 month after disease remission.

This study was approved by the institutional review board of Kurume University School of Medicine, and complied with the Helsinki Declaration. 


\subsection{Immunohistochemistry}

The sections stained with hematoxylin and eosin (H \& E) and the available immunohistochemical slides of all cases were re-examined. Immunohistochemical analyses were performed using $2-\mu \mathrm{m}$ sections by standard immunohistochemistry methods. Antibodies against the following antigens were utilized: CD5 (clone 4C7; Novocastra, Newcastle, UK), CD10 (clone 56C6; Novocastra), BCL6 (clone P1F6; Novocastra), multiple myeloma-1/interferon regulatory factor-4 (MUM1/IRF4, clone MUM1p; Dako, Glostrup, Denmark), and Ki67 (clone MIB 1; Dako). The antibodies were visualized with the ChemMate ENVISION method (Dako). Diaminobenzidine (DAB) was used as a substrate. Sections were counterstained by hematoxylin.

\subsection{Evaluation of Immunostained Sections}

Immunoreactivity was determined without any knowledge of survival or other clinical data. A minimum of 500 tumor cells were evaluated in each of $3-5$ microscopic fields. All antigen expression except that of Ki67 was evaluated by assessing the percentage of immunoreactive tumor cells in $10 \%$ increments. Ki67 expression was evaluated by assessing the percentage of immunoreactive tumor cells in 5\% increments. For evaluation of CD5, CD10, BCL6, and MUM1/IRF4, more than 30\% positivity of the tumor cells was judged to indicate positivity for the purposes of this study. We determined the evaluation method of the expression of the antibodies by referring to the previous reports $[15,23,29]$, and by considering the method used clinically.

\subsection{Classification of Tumors as GCB or Non-GCB}

We used the immunohistochemical expression of CD10, BCL6, or MUM1/IRF4 to classify cases of DLBCL into GCB and non-GCB groups according to the Hans algorithm (Figure 1) [15].

\subsection{Statistical Analysis}

Clinical characteristics of the 2 groups (GCB vs nonGCB, CD5+ DLBCL vs CD5- DLBCL) were compared

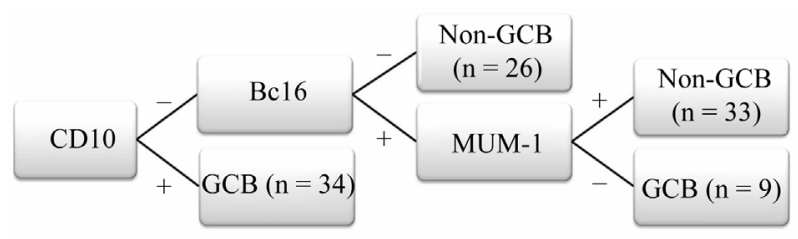

Figure 1. Decision tree for immunohistochemical classification of diffuse large B-cell lymphoma (germinal center Bcell $[\mathrm{GCB}]$ or non-GCB). using the Mann-Whitney $U$ test or the chi-square test. We used cause-specific survival (CSS) and relapse-free rate (RFR) to compare the prognosis of the patients. Because all cases that were deceased due to other causes were part of the GCB group (Table 1). CSS was defined as the time from initial diagnosis to the date of death or last contact. RFR was defined as the time from initial diagnosis to the date of relapse. CSS and RFR curves were estimated by the Kaplan-Meier method. Statistical differences between these curves were determined using the log-rank test for each of the parameters. Multivariate analysis was performed using the Cox proportional hazard regression model to compare CSS and RFR distributions following adjustment for the variables mentioned. A $p$ value of less than 0.05 was considered statistically significant. The analysis was carried out using JMP 10 for Windows (SAS, Cary, NC, USA).

\section{Results}

\subsection{Patient Characteristics}

This study included 102 patients (53 men [52\%] and 49 women [48\%]); the median age of the patients was 68 years (range, 21 to 88 years). According to the Ann Arbor classification, 36 cases were at clinical stage I and 66 at stage II. Seven patients had a B-ALPS of 0,28 had a B-ALPS of 1, 28 had a B-ALPS of 2, 22 had a B-ALPS of 3,13 had a B-ALPS of 4, and 4 had a B-ALPS of 5 . Twenty-three patients received radiotherapy alone, whereas 79 patients were treated with radiotherapy and chemotherapy. The chemotherapy regimens used were CHOP or CHOP-like regimens, with or without rituximab. Fortytwo patients received a CHOP-like regimen, and 37 patients received an R-CHOP-like regimen. The median follow-up of surviving patients was 52 months (range, 3 to 138 months).

At the time of analysis, 19 patients (19\%) had died, and 83 patients $(81 \%)$ were alive at last contact. Thirteen patients died as a result of malignant lymphoma, and 6 due to other disease. Twenty-three relapses occurred, at abdominal LNs (6 cases), multiple sites (6 cases), central nervous system (4 cases), axillary LNs ( 2 cases), ileum end ( 1 case), stomach (1 case), and testis ( 1 case), and only 2 relapses occurred inside the previous radiotherapy field. The characteristics of the patients with respect to age, sex, stage, B-ALPS, and treatment are listed in Table 2. The 2-year CSS rate of all 102 patients, estimated by the Kaplan-Meier method was $88.5 \%$, and the 5 -year overall survival rate was $86.8 \%$. The 2 -year RFR for the entire group was $80.9 \%$ and the 5 -year RFR was $79.4 \%$.

\subsection{Immunochemical Subtypes}

According to the Hans algorithm, 43 cases (42\%) were 
Table 1. Patient characteristics: dead cases with other causes.

\begin{tabular}{ccclccc}
\hline No. & Age & Gender & Cause for death & Chemotherapy & Month after diagnosis & GCB or non GCB \\
\hline 1 & 88 & F & Ileus & - & 3 & GCB \\
2 & 73 & M & Cerebral inferction & R-CHOP & 6 & GCB \\
3 & 86 & F & Cerebral inferction & - & 44 & GCB \\
4 & 79 & M & Senility & T-COP & 74 & GCB \\
5 & 74 & F & Inflectional colitis & CHOP & 95 & GCB \\
6 & 68 & F & Breast cancer & GCB \\
\hline
\end{tabular}

Abbrebiations: GCB, germinal B-cell; R, rituximab; CHOP, cyclophosphamide, doxotubicin, vincristine, prednisone; T-COP, tetrahydropyranyl adriamycin, cyclophosphamide, doxotubicin, prednisone.

Table 2. Patient characteristics.

\begin{tabular}{|c|c|c|c|c|c|c|c|}
\hline & Total & GCB & non GCB & $\mathrm{p}$ value & CD5+ & CD5- & $\mathrm{p}$ value \\
\hline No. of patients & 102 & 43 & 59 & & 33 & 69 & \\
\hline Gender & & & & 0.89 & & & 0.363 \\
\hline Male & 53 & 22 & 31 & & 15 & 39 & \\
\hline Female & 49 & 21 & 28 & & 19 & 30 & \\
\hline Age at diagnosis median (range) & $68(21-88)$ & $68(29-88)$ & $69(21-88)$ & 0.919 & $70(38-88)$ & $68(21-88)$ & 0.9 \\
\hline Ann Arbor stage & & & & 0.183 & & & 0.466 \\
\hline I & 36 & 12 & 24 & & 10 & 26 & \\
\hline II & 66 & 31 & 35 & & 23 & 43 & \\
\hline B-ALPS & & & & 0.979 & & & 0.883 \\
\hline 0 & 7 & 3 & 4 & & 3 & 4 & \\
\hline 1 & 27 & 10 & 17 & & 9 & 19 & \\
\hline 2 & 31 & 17 & 16 & & 10 & 18 & \\
\hline 3 & 20 & 8 & 12 & & 5 & 17 & \\
\hline 4 & 13 & 6 & 7 & & 5 & 8 & \\
\hline 5 & 4 & 1 & 3 & & 1 & 3 & \\
\hline Chemotherapy & & & & 0.289 & & & 0.435 \\
\hline No chemotherapy & 23 & 8 & 15 & & 5 & 18 & \\
\hline CHOP-like regimen & 42 & 22 & 20 & & 16 & 27 & \\
\hline R-CHOP-like regimen & 37 & 13 & 24 & & 12 & 24 & \\
\hline Follow-up median (range) & $52(3-138)$ & $57(3-138)$ & $49(7-135)$ & 0.483 & $62(11-135)$ & $33(2-138)$ & 0.069 \\
\hline
\end{tabular}

Abbrebiations: GCB, germinal B-cell; B-ALPS, prognostic index for early stage non-Hodgkin lymphoma; R, rituximab; CHOP, cyclophosphamide, doxotubicin, vincristine, prednisone.

considered GCB and 59 cases (58\%) non-GCB. Examples of immunohistochemical subclassification are shown in Figure 2. The clinical features of the patients with immunohistochemistry of GCB and non-GCB are shown in Table 2. The clinical features were not significantly different between the 2 subtypes. The median follow-up for the GCB and the non-GCB patients was 57 months (range, 3 to 138 months) and 49 months (range, 7 to 135 months). The 5-year CSS and RFR for the GCB group were 92.4\% and $90.2 \%$ compared with $82.8 \%$ and $71.9 \%$ for the
non-GCB group, respectively. There were $33 \mathrm{CD} 5+$ cases (32\%), and 69 CD5- cases (68\%). The median follow-up for the CD5+ and CD5- patients was 74 months (range, 11 to 135 months) and 73 months (range, 3 to 138 months), respectively. The clinical features of the CD5+ and CD5- patients are shown in Table 2; they were not significantly different between the 2 subtypes. The 5 year CSS and RFR for the CD5+ group were $88.9 \%$ and $86.8 \%$ compared with $85.9 \%$ and $76 \%$ for the CD5group, respectively. 


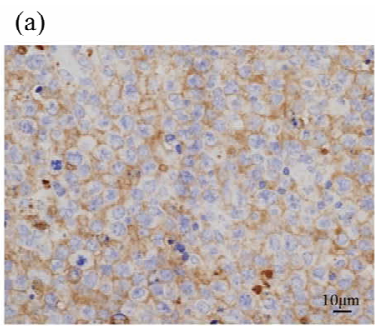

CD10

(b)

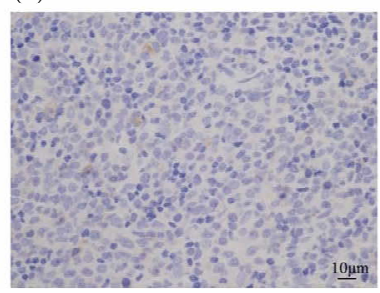

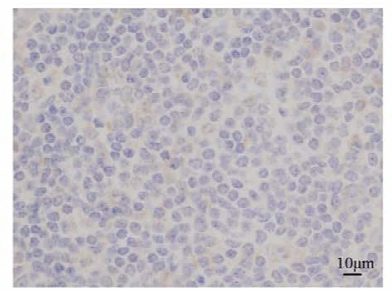

bc16

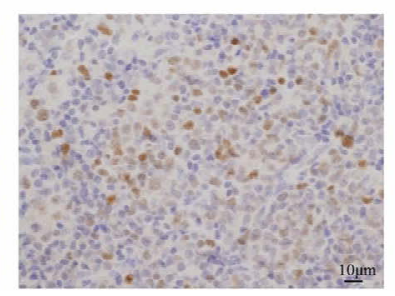

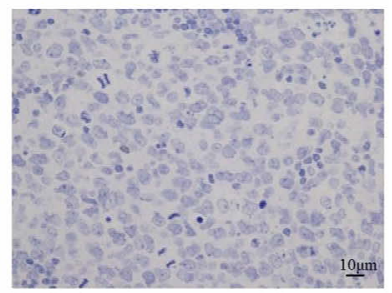

MUM1

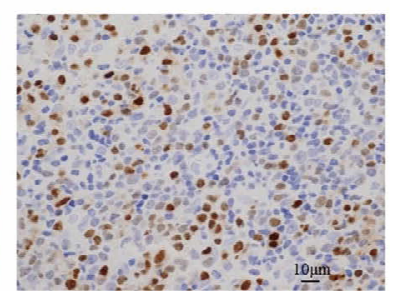

Figure 2. Results of immunohistochemical staining. (a) Immunohistochemical stains of a germinal center B-cell (GCB) case that is positive for CD10 but negative for BCL6 and MUM1/IRF4; (b) Immunohistochemical stains of a non-GCB case that is negative for CD10 but positive for BCL6 and MUM1/IRF4. Original magnification, $\times 400$.

\subsection{Prognostic Factor Analyses}

Variables considered in the analysis were the cell of origin classification (GCB or non-GCB), B-ALPS, CD5, Ki67, chemotherapy regimen (no chemotherapy, CHOPlike regimen, and R-CHOP-like regimen), and sex. According to B-ALPS, patients were divided into 3 groups: the low risk group had risk factors from 0 to 1 , the intermediate group had risk factors of 2 , and the high-risk group had risk factors from 3 to 5 . In univariate analysis, the cell of origin classification was a significant factor influencing RFR ( $\mathrm{p}=0.033$ ) (Figure 3(a)), but none of these variables were significant for CSS. Multivariate analysis was performed using information from all of the variables. The cell of origin classification was an independent predictor of CSS $(p=0.040)$, with those in the non-GCB group having a 3.71-fold (95\% CI: 1.06 - 17.6) greater risk of death (Figure 3(b)). This classification was the only independent predictor of RFR $(p=0.023)$, with those in the non-GCB group having a 2.96-fold (95\% CI: 1.15 - 9.12) greater risk of relapse (Figure 3(a)). The chemotherapy regimen was also an independent predictor of CSS, with cases who had no chemotherapy having a 11.9 -fold $(95 \% \mathrm{CI}: 1.50-253, \mathrm{p}=$ 0.018) greater risk of death than those who had the RCHOP-like regimen. Cases with a CHOP-like regimen had a 7.68 -fold $(95 \% \mathrm{CI}: 1.31-146, \mathrm{p}=0.021)$ greater risk of death than those who had a R-CHOP-like regimen. However, in the cases treated without rituximab, there were no statistically significant differences between cases with no chemotherapy (radiotherapy alone) and those who received a CHOP-like regimen (Figure 4(a)). This result implies that addition of rituximab to the radiother- apy treatment or CHOP-like chemotherapy improves prognosis. The data are listed in Table 3.

We divided the patients into the GCB group and nonGCB group, and analyzed the statistics separately. In the GCB group, no independent predictors of CSS and RFR were found. On the other hand, in the non-GCB group the chemotherapy regimen was an independent predictor of CSS, with no chemotherapy cases having a 13.0-fold (95\% CI: $1.25-308, p=0.031$ ) greater risk of death than those who had a R-CHOP-like regimen. In addition, the CHOP-like regimen resulted in a 9.22-fold (95\% CI: 1.44 $-180, p=0.017)$ greater risk of death than the R-CHOPlike regimen. There was no statistically significant difference between the radiotherapy alone cases and those who had a CHOP-like regimen without rituximab (Figure $4(b))$.

\section{Discussion}

DLBCL is heterogeneous with respect to clinical presentation, genetic features, and the cell of origin [30,31]. In an attempt to elucidate the underlying factors contributing to this disease, the prognostic value of numerous individual proteins has been studied by immunohistochemical and molecular techniques [7,8,32-34]. The expression of individual antigens related to the different stages of B-cell differentiation, including CD10, BCL6, MUM1/ IRF4, and CD5 may help to define groups of tumors with different clinical and pathological characteristics $[10,11$, 13].

The distinction of the GCB and non-GCB subtypes is beginning to translate into the clinic, as these diagnostic categories have significantly different survival rates after 
Table 3. The result of Cox's multivariate proportional hazards analysis of prognostic factor cause specific survival.

\begin{tabular}{|c|c|c|c|c|}
\hline \multirow[b]{2}{*}{ Valiable } & \multicolumn{2}{|c|}{ Cause specific survival } & \multicolumn{2}{|c|}{ Relapse free rate } \\
\hline & $\mathrm{p}$ value & Hazard ratio $(95 \% \mathrm{CI})$ & $\mathrm{p}$ value & Hazard ratio $(95 \% \mathrm{CI})$ \\
\hline \multicolumn{5}{|l|}{ Gender } \\
\hline male vs female & 0.241 & $2.04(0.630-7.87)$ & 0.397 & $1.45(0.614-3.62)$ \\
\hline \multicolumn{5}{|l|}{ B-ALPS } \\
\hline low vs intermediate & 0.946 & $0.956(0.262-3.62)$ & 0.787 & $0.868(0.308-2.47)$ \\
\hline intermediate vs high & 0.134 & $3.37(0.696-24.2)$ & 0.374 & $1.64(0.550-5.13)$ \\
\hline high vs low & 0.133 & $0.311(0.044-1.40)$ & 0.515 & $0.704(0.229-2.03)$ \\
\hline \multicolumn{5}{|l|}{ GCB or non-GCB } \\
\hline non $\mathrm{GCB}$ vs $\mathrm{GCB}$ & 0.04 & $3.71(1.06-17.6)$ & 0.023 & $2.96(1.15-9.12)$ \\
\hline \multicolumn{5}{|l|}{ Chemotherapy (CTx) } \\
\hline $\mathrm{RT}$ alone (no CTx) vs CHOP-like regimen & 0.055 & $1.55(0.347-6.28)$ & 0.602 & $1.34(0.431-4.06)$ \\
\hline RT alone vs R-CHOP-like regimen & 0.018 & $11.9(1.50-253)$ & 0.095 & $2.85(0.832-10.6)$ \\
\hline CHOP-like regimen vs R-CHOP-like regimen & 0.02 & $7.68(1.32-146)$ & 0.173 & $2.12(0.725-7.00)$ \\
\hline \multicolumn{5}{|l|}{ CD5 } \\
\hline$(+)$ vs $(-)$ & 0.717 & $0.803(0.212-2.53)$ & 0.219 & $0.548(0.179-1.40)$ \\
\hline Ki67 & 0.113 & $0.976(0.948-1.01)$ & 0.87 & $0.991(0.888-1.11)$ \\
\hline
\end{tabular}

Abbreviations: CI, confidence interval; B-ALPS, prognostic index for early stage non-Hodgkin lymphoma; GCB, germinal B-cell; RT, radiotherapy; R, rituximab; CHOP, cyclophosphamide, doxotubicin, vincristine, prednisone.

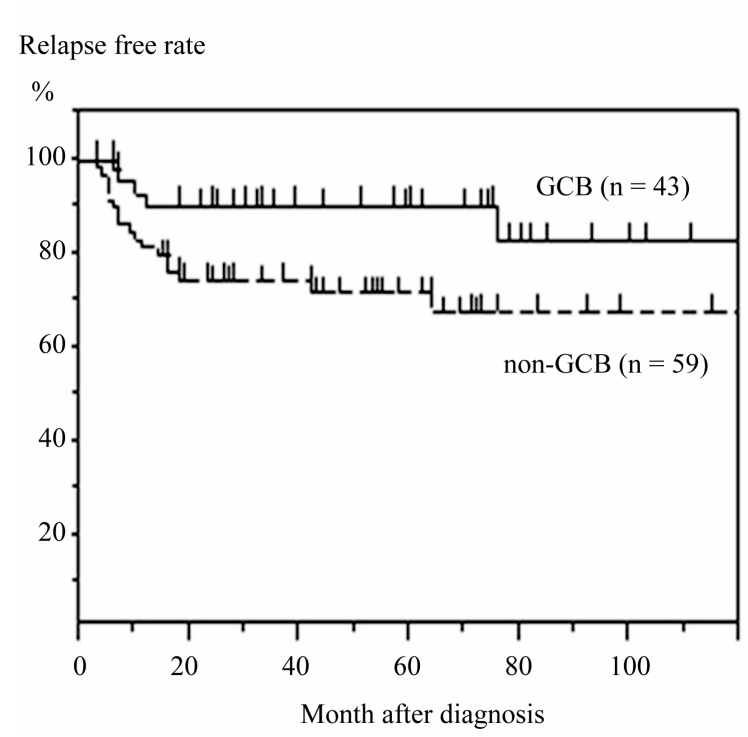

(a)

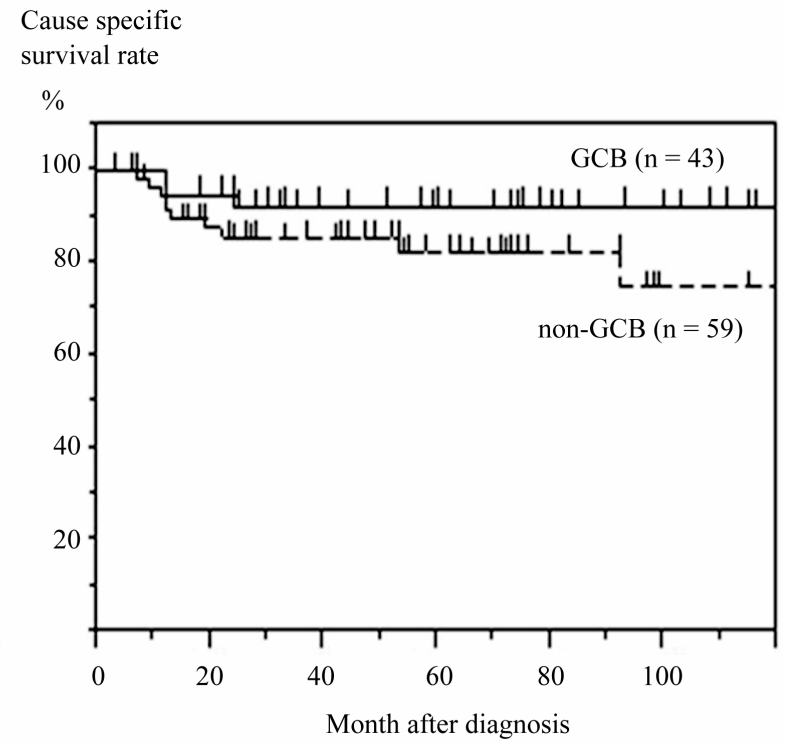

(b)

Figure 3. (a) Relapse-free rate (RFR) curves and the cell of origin classification. RFR curves using the immunohistochemical classification of germinal center B-cell (GCB) versus non-GCB. Statistically significant differences were found between GCB and non-GCB $(p=0.033$, log-rank test) (hazard ratio for relapse, 2.96 [95\% CI, $1.15-9.12] ; p=0.023$. Cox proportional hazard regression model); (b) Cause-specific survival (CSS) curves and the cell of origin classification. CSS curves using the immunohistochemical classification of germinal center B-cell (GCB) versus non-GCB. In multivariate analysis, statistically significant differences were found between GCB and non-GCB (hazard ratio for death, 3.71 [95\% CI, 1.06 - 17.6]; $p=0.040$. Cox proportional hazard regression model). 


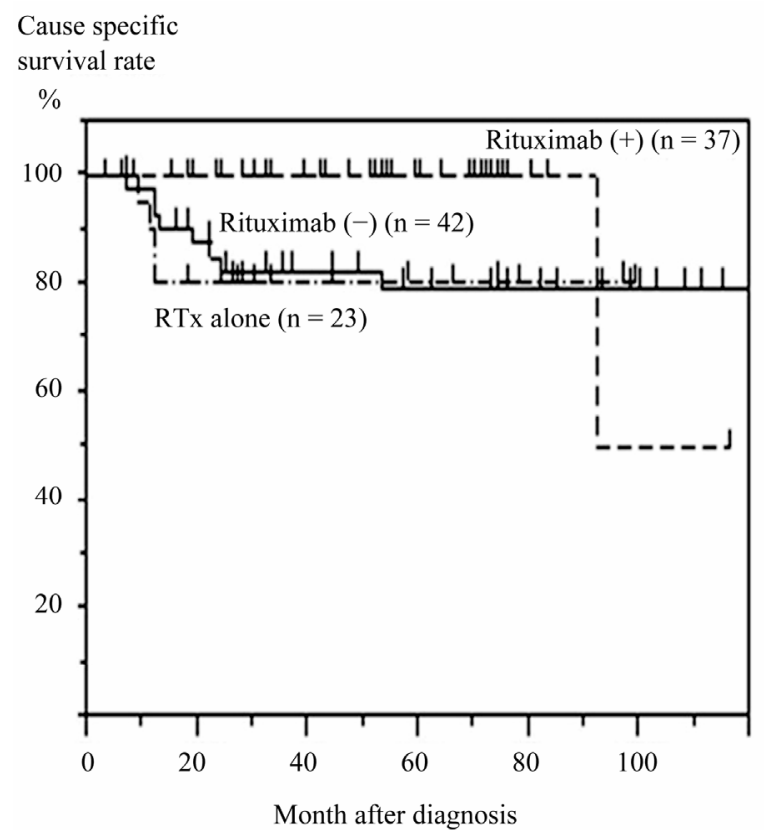

(a)
Cause specific

survival rate

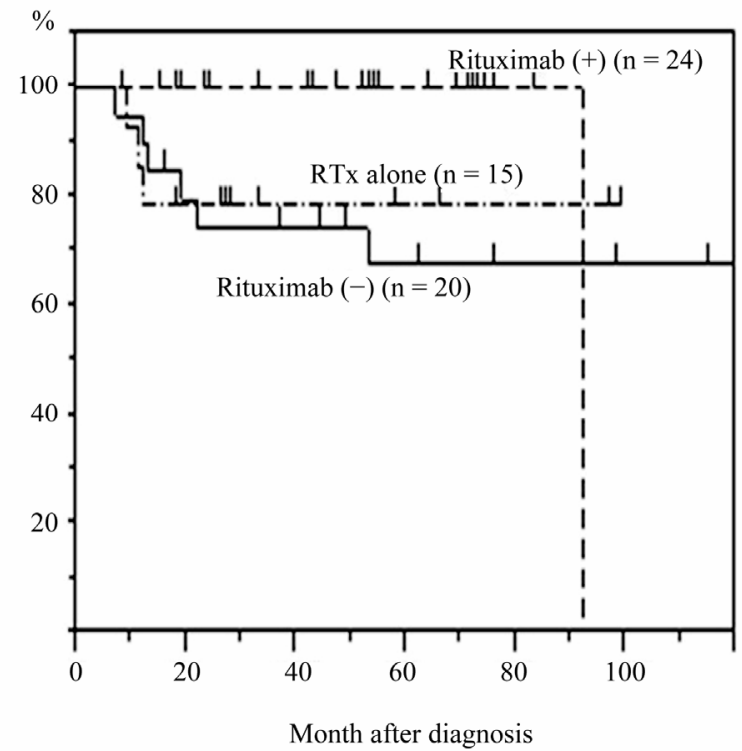

(b)

Figure 4. (a) Cause-specific survival (CSS) curves and the regimen of chemotherapy. CSS curves using radiatiotherapy (RT) alone (no chemotherapy) vs radiotherapy + CHOP-like regimen (Rituximab $(-)$ ) vs radiotherapy + R-CHOP-like regimen (Rituximab $(+)$ ). In multivariate analysis, statistically significant differences were found between radiotherapy alone and radiotherapy + R-CHOP-like regimen (hazard ratio for death, 13.0 [95\% CI, $1.25-308], p=0.031$. Cox proportional hazard regression model). Statistically significant differences were also shown between radiotherapy $+\mathrm{CHOP-like} \mathrm{regimen} \mathrm{and} \mathrm{ra-}$ diotherapy + R-CHOP-like regimen (hazard ratio for death, 9.22 [95\% CI, $1.44-180]$; $p=0.017$. Cox proportional hazard regression model). No significant differences were shown between radiotherapy alone and radiotherapy $+\mathrm{CHOP}-$ like regimen, statistically; (b) Cause-specific survival (CSS) curves and the regimen of chemotherapy in non-GCB group. CSS curves using radiotherapy (RT) alone (no chemotherapy) vs radiotherapy + CHOP-like regimen (Rituximab $[-])$ vs radiotherapy + R-CHOP-like regimen $($ Rituximab $[+])$. In multivariate analysis, statistically significant differences were found between radiotherapy alone and radiotherapy + R-CHOP-like regimen (hazard ratio for death, $11.9[95 \% \mathrm{CI}, 1.50-253], \mathrm{p}=0.018 . \mathrm{Cox}$ proportional hazard regression model). Statistically significant differences were also shown between radiotherapy $+\mathrm{CHOP}-$ like regimen and radiotherapy + R-CHOP-like regimen (hazard ratio for death, $7.68[95 \% \mathrm{CI}, 1.32-146] ; \mathbf{p}=0.020$. Cox proportional hazard regression model). No significant differences were shown between radiotherapy alone and radiotherapy + CHOP-like regimen, statistically.

standard treatment. A few studies have used the immunohistochemical expression of CD10, BCL6, or MUM1/ IRF4 to classify cases of DLBCL into GCB and nonGCB groups [11,34]. Various studies have shown that this classification on the basis of the cell of origin is an important independent prognostic factor for patients who are treated with CHOP or CHOP-like chemotherapy [1517]. However, in the rituximab era, the resulting data have been controversial, with several studies showing a significantly better survival rate for the GCB group and others finding no difference in survival between the GCB and non-GCB groups $[15,34,35]$. With regard to the association of the GCB and non-GCB subtypes, and the expression of Ki67, it has been reported that prognosis is poorest for patients with elevated Ki67 expression in the non-GCB subgroup [25].

De novo CD5+ DLBCL is a subtype of DLBCL with a poor clinical outcome. Yamaguchi et al. reported that its prognosis was significantly poorer than that of CD5tumors [12]. In contrast, it has also been reported that the expression of CD5 in DLBCL did not affect overall survival [36].

Radiotherapy is effective for malignant lymphoma, and some reports have suggested that localized NHL may be successfully treated with radiotherapy alone until rituximab era $[26,27]$. So we especially focused localizedstage head and neck DLBCL treated with curative radiotherapy. To our knowledge, an analysis of the relationship between classification on the basis of the cell of origin or expression of CD5 and the prognosis of patients with localized-stage head and neck DLBCL, treated with curative radiotherapy with or without chemotherapy, has not been reported. In this study, we investigated the clinical significance and prognostic value of different immunophenotypic profiles related to B-cell differentiation, CD5 and Ki67 expression in patients with localized-stage 
head and neck DLBCL treated with curative radiotherapy.

We found that the prognosis of the GCB subtype was significantly better than the non-GCB subtype in localized-stage head and neck DLBCL, which indicates curative radiotherapy. This classification, on the basis of the cell of origin, is predictive of survival in patients with DLBCL who were treated with curative radiotherapy with or without chemotherapy. Our findings confirm previous studies showing that the patients with an immunohistochemically-defined GCB phenotype have a significantly better outcome compared to patients with a nonGCB phenotype [15-17]. Therefore combining these biomarkers may provide further prognostic information in addition to that yielded by the clinical index. We found no correlation between the expression of CD5 or Ki67 in DLBCL patients and the survival or relapse rate.

Rituximab is a monoclonal antibody targeting CD20, a common B-cell marker that is present on the majority of malignant lymphoma cells. Some groups have demonstrated a survival benefit from the addition of rituximab to CHOP [37]. Thus, rituximab continues to be an integral component of treatment for DLBCL patients. We found that the addition of rituximab to radiotherapy or standard chemotherapy improved the survival of patients with localized-stage head and neck DLBCL in non-GCB group, but in GCB group. This study confirms the advantage of a combined radiotherapy-immunochemotherapy approach for these patients. In cases involving elderly people, it is sometimes difficult to add chemotherapy due to their complications, and it may be preferable to just add rituximab to curative radiotherapy. The further research is needed.

Recent clinical studies suggest that the prognosis of the non-GCB subgroup of DLBCL is improved by rituximab-containing chemotherapy $[18,38,39]$. In this study, we also found that the addition of rituximab improved CSS in the non-GCB group, and this result reached statistical significance, but this was not the case for the GCB group. Therefore, the addition of rituximab should be considered an important part of treatment for the nonGCB subgroup of DLBCL patients.

However, there are some limitations to this study as it was a retrospective one, and there may be a selection bias related to the type of therapy employed, either for the group that received radiotherapy alone or for the group that received additional chemotherapy. Moreover, the number of patients analyzed was not large. Despite these limitations, to our knowledge, this is the first study to report that the cell of origin classification predicts prognosis in patients with localized-stage DLBCL, all of whom received curative radiotherapy.

In conclusion, the present study shows that the prog- nosis of the non-GCB group was significantly poorer than that of the GCB group in patients with localizedstage head and neck DLBCL. In addition, we showed that rituximab improved the CSS in localized-stage nonGCB DLBCL. We may be able to refer to this immunohistochemical subclassification when deciding future treatment plans for patients with localized-stage DLBCL.

\section{REFERENCES}

[1] B. Coiffier, "Diffuse Large Cell Lymphoma," Current Opinion in Oncology, Vol. 13, No. 5, 2001, pp. 325-334. doi:10.1097/00001622-200109000-00003

[2] The Non-Hodgkin's Lymphoma Classification Project, "A Clinical Evaluation of the International Lymphoma Study Group classification of Non-Hodgkin's lymphoma," Blood, Vol. 89, No. 11, 1997, pp. 3909-3918.

[3] N. L. Harris, E. S. Jaffe, H. Stein, et al., “A Revised European-American Classification of Lymphoid Neoplasms: A Proposal from the International Lymphoma Study Group," Blood, Vol. 84, No. 5, 1994, pp. 1361-1392.

[4] The International Non-Hodgkin's Lymphoma Prognostic Factors Project, "A Predictive Model for Aggressive NonHodgkin's Lymphoma," New England Journal of Medicine, Vol. 329, No. 14, 1993, pp. 987-994. doi:10.1056/NEJM199309303291402

[5] A. Rosenwald, G. Wright, W. C. Chan, et al., "The Use of Molecular Profiling to Predict Survival after Chemotherapy for Diffuse Large-B-Cell Lymphoma," New England Journal of Medicine, Vol. 346, No. 25, 2002, pp. 1937-1947. doi:10.1056/NEJMoa012914

[6] M. A. Shipp, K. N. Ross, P. Tamayo, et al., "Diffuse Large B-Cell Lymphoma Outcome Prediction by Gene-Expression Profiling and Supervised Machine Learning," Nature Medicine, Vol. 8. No. 1, 2002, pp. 68-74. doi:10.1038/nm0102-68

[7] S. Harada, R. Suzuki, K. Uehira, et al., "Molecular and Immunological Dissection of Diffuse Large B Cell Lymphoma: CD5+, and CD5- with CD10+ Groups May Constitute Clinically Relevant Subtypes," Leukemia, Vol. 13, No. 9, 1999, pp. 1441-1447. doi:10.1038/sj.leu.2401487

[8] K. Ohshima, C. Kawasaki, H. Muta, et al., "CD10 and Bcl10 Expression in Diffuse Large B-Cell Lymphoma: CD10 is a Marker of Improved Prognosis," Histopathology, Vol. 39, No. 2, 2001, pp. 156-162. doi:10.1046/j.1365-2559.2001.01196.x

[9] A. A. Alizadeh, M. B. Eisen, R. E. Davis, et al., "Distinct Types of Diffuse Large B-Cell Lymphoma Identified by Gene Expression Profiling," Nature, Vol. 403, No. 6769, 2000, pp. 503-511. doi:10.1038/35000501

[10] B. E. King, C. Chen, J. Locker, et al., "Immunophenotypic and Genotypic Markers of Follicular Center Cell Neoplasia in Diffuse Large B-Cell Lymphoma," Modern Pathology, Vol. 13, No. 10, 2000, pp. 1219-1231. doi:10.1038/modpathol.3880226

[11] A. Dogan, E. Bagdi, P. Munson and P. G. Isaacson, 
"CD10 and BCL-6 Expression in Paraffin Sections of Normal Lymphoid Tissue and B-Cell Lymphomas," American Journal of Surgical Pathology, Vol. 24, No. 6, 2000, pp. 846-852.

doi:10.1097/00000478-200006000-00010

[12] M. Yamaguchi, M. Seto, M. Okamoto, et al., "De Novo CD5+ Diffuse Large B-Cell Lymphoma: A Clinicopathologic Study of 109 Patients," Blood, Vol. 99, No. 3, 2002, pp. 815-821. doi:10.1182/blood.V99.3.815

[13] H. J. Ree, W. I. Yang, C. W. Kim, et al., "Coexpression of Bcl-6 and CD10 in Diffuse Large B-Cell Lymphomas: Significance of Bcl-6 Expression Patterns in Identifying Germinal Center B-Cell Lymphoma," Human Pathology, Vol. 32, No. 9, 2001, pp. 954-962. doi:10.1053/hupa.2001.27118

[14] G. Lenz, G. Wright, S. S. Dave, et al., "Stromal Gene Signatures in Large-B-Cell Lymphomas," New England Journal of Medicine, Vol. 359, No. 22, 2008, pp. 23132323. doi:10.1056/NEJMoa0802885

[15] C. P. Hans, D. D. Weisenburger, T. C. Greiner, et al., "Confirmation of the Molecular Classification of Diffuse Large B-Cell Lymphoma by Immunohistochemistry Using a Tissue Microarray," Blood, Vol. 13, No. 1, 2004, pp. 275-282. doi:10.1182/blood-2003-05-1545

[16] M. Berglund, U. Thunberg, R. M. Amini, et al., "Evaluation of Immunophenotype in Diffuse Large B-Cell Lymphoma and Its Impact on Prognosis," Modern Pathology, Vol. 18, No. 8, 2005, pp. 1113-1120.

doi:10.1038/modpathol.3800396

[17] G. W. van Imhoff, E. J. Boerma, B. van der Holt, et al., "Prognostic Impact of Germinal Center-Associated Proteins and Chromosomal Breakpoints in Poor-Risk Diffuse Large B-Cell Lymphoma," Journal of Clinical Oncology, Vol. 24, No. 25, 2006, pp. 4135-4142. doi:10.1200/JCO.2006.05.5897

[18] H. Nyman, M. Adde, M. L. Karjalainen-Lindsberg, et al., "Prognostic Impact of Immunohistochemically Defined Germinal Center Phenotype in Diffuse Large B-Cell Lymphoma Patients Treated with Immunochemotherapy," Blood, Vol. 109, No. 11, 2007, pp. 4930-4935. doi:10.1182/blood-2006-09-047068

[19] R. Seki, K. Ohshima, T. Fujisaki, et al., "Prognostic Impact of Immunohistochemical Biomarkers in Diffuse Large B-Cell Lymphoma in the Rituximab Era," Cancer Science, Vol. 100, No. 10, 2009, pp. 1842-1847. doi:10.1111/j.1349-7006.2009.01268.x

[20] K. Fu, D. D. Weisenburger, W. W. Choi, et al., "Addition of Rituximab to Standard Chemotherapy Improves the Survival of Both the Germinal Center B-Cell-Like and Non-Germinal Center B-Cell-Like Subtypes of Diffuse Large B-Cell Lymphoma," Journal of Clinical Oncology, Vol. 26, No. 28, 2008, pp. 4587-4594. doi:10.1200/JCO.2007.15.9277

[21] D. C. Brown and K. C. Gatter, "Ki67 Protein: The Immaculate Deception?" Histopathology, Vol. 40, No. 1, 2002, pp. 2-11. doi:10.1046/j.1365-2559.2002.01343.x

[22] T. Scholzen and J. Gerdes, "The Ki-67 Protein: From the Known and the Unknown," Journal of Cellular Physiol- ogy, Vol. 182, No. 3, 2000, pp. 311-332. doi:10.1002/(SICI)1097-4652(200003)182:3<311::AID-J CP1>3.0.CO;2-9

[23] K. Szczuraszek, G. Mazur, M. Jelen, P. Dziegiel, P. Surowiak and M. Zabel, "Prognostic Significance of Ki-67 Antigen Expression in Non-Hodgkin's Lymphomas," Anticancer Research, Vol. 28, No. 2A, 2008, pp. 11131118.

[24] P. Dziegiel, W. Salwa-Zurawska, J. Zurawski, A. Wojnar and M. Zabel, "Prognostic Significance of Augmented Metallothionein (MT) Expression Correlated with Ki-67 Antigen Expression in Selected Soft Tissue Sarcomas," Histology and Histopathology, Vol. 20, No. 1, 2005, pp. 83-89. doi:10.1111/j.1600-0609.2012.01778.x

[25] Z. M. Li, J. J. Huang, Y. Xia, et al., "High Ki-67 Expression in Diffuse Large B-Cell Lymphoma Patients with Non-Germinal Center Subtype Indicates Limited Survival Benefit from R-CHOP Therapy," European Journal of Haematology, Vol. 88, No. 6, 2012, pp. 510-517.

[26] N. Hayabuchi, Y. Shibamoto, K. Nakamura, et al., "Stage I and II Aggressive B-Cell Lymphomas of the Head and Neck: Radiotherapy Alone as a Treatment Option and the Usefulness of the New Prognostic Index B-ALPS," International Journal of Radiation Oncology Biology Physics, Vol. 55, No. 1, 2003, pp. 44-50. doi:10.1016/S0360-3016(02)03798-7

[27] K. Sunaba, H. Shibuya, N. Okada, T. Amagasa, S. Enomoto and S. Kishimoto "Radiotherapy for Primary Localized (Stage I and II) Non-Hodgkin's Lymphoma of the Oral Cavity," International Journal of Radiation Oncology Biology Physics, Vol. 47, No. 1, 2000, pp. 179-183. doi:10.1016/S0360-3016(00)00412-0

[28] M. Oguchi, H. Ikeda, K. Isobe, et al., "Tumor Bulk as a Prognostic Factor for the Management of Localized Aggressive Non-Hodgkin's Lymphoma: A Survey of the Japan Lymphoma Radiation Therapy Group," International Journal of Radiation Oncology Biology Physics, Vol. 48, No. 1, 2000, pp. 161-168.

doi:10.1016/S0360-3016(00)00480-6

[29] M. Yamaguchi, N. Nakamura, R. Suzuki, et al., "De novo CD5+ Diffuse Large B-Cell Lymphoma: Results of a Detailed Clinicopathological Review in 120 Patients," $\mathrm{He}$ matologica, Vol. 93, No. 8, 2008, pp. 1195-1202. doi: $10.3324 /$ haematol.12810

[30] S. Monti, K. J. Savage, J. L. Kutok, et al., "Molecular Profiling of Diffuse Large B-Cell Lymphoma Identifies Robust Subtypes Including One Characterized by Host Inflammatory Response," Blood, Vol. 105, No. 5, 2005, pp. 1851-1861. doi:10.1182/blood-2004-07-2947

[31] C. Adida, C. Haioun, P. Gaulard, et al., "Prognostic Significance of Surviving Expression in Diffuse Large BCell Lymphomas,” Blood, Vol. 96, No. 5, 2000, pp. 19211925.

[32] A. Zhang, K. Ohshima, K. Sato, et al., "Prognostic Clinicopathologic Factors, Including Immunologic Expression in Diffuse Large B-Cell Lymphomas," Pathology International, Vol. 49, No. 12, 1999, pp. 1043-1052. doi:10.1046/j.1440-1827.1999.00980.x 
[33] M. Takeshita, A. Iwashita, K. Kurihara, et al., "Histologic and Immunohistologic Findings and Prognosis of 40 Cases of Gastric Large B-Cell Lymphoma," The American Journal of Surgical Pathology, Vol. 24, No. 12, 2000, pp. 1641-1649. doi:10.1097/00000478-200012000-00008

[34] L. Colomo, A. López-Guillermo, M. Perales, et al., "Clinical Impact of the Differentiation Profile Assessed by Immunophenotyping in Patients with Diffuse Large BCell Lymphoma," Blood, Vol. 101, No. 1, 2003, pp. 7884. doi:10.1182/blood-2002-04-1286

[35] C. C. Chang, S. McClintock, R. P. Cleveland, et al., "Immunohistochemical Expression Patterns of Germinal Center and Activation B-Cell Markers Correlate with Prognosis in Diffuse Large B-Cell Lymphoma," The American Journal of Surgical Pathology, Vol. 28, No. 4, 2004, pp. 464-470. doi:10.1097/00000478-200404000-00005

[36] T. Katzenberger, A. Lohr, S. Schwarz, et al., "Genetic Analysis of de Novo CD5+ Diffuse Large B-Cell Lymphomas Suggests an Origin from a Somatically Mutated
CD5+ Progenitor B Cell,” Blood, Vol. 101, No. 2, 2003, pp. 699-702. doi:10.1182/blood-2002-06-1726

[37] P. Feugier, A. Van Hoof, C. Sebban, et al., "Long-Term Results of the R-CHOP Study in the Treatment of Elderly Patients with Diffuse Large B-Cell Lymphoma: A Study by the Groupe d'Etude des Lymphomes de l'Adulte," Journal of Clinical Oncology, Vol. 23, No. 18, 2005, pp. 4117-4126. doi:10.1200/JCO.2005.09.131

[38] J. N. Winter, E. A. Weller, S. J. Horning, et al., "Prognostic Significance of Bcl-6 Protein Expression in DLBCL Treated with CHOP or R-CHOP: A Prospective Correlative Study," Blood, Vol. 107, No. 11, 2006, pp. 4207 4213. doi:10.1182/blood-2005-10-4222

[39] N. Mounier, J. Briere, C. Gisselbrecht, et al., "Rituximab Plus CHOP (R-CHOP) Overcomes bcl-2-Associated Resistance to Chemotherapy in Elderly Patients with Diffuse Large B-Cell Lymphoma (DLBCL)," Blood, Vol. 101, No. 11, 2003, pp. 4279-4284. doi:10.1182/blood-2002-11-3442 